\title{
Knockdown of astrocyte elevated gene- 1 inhibits growth through suppression of IL-6 secretion in HepG2 human hepatoma cells
}

\author{
HUAN DENG, ZHENZHEN ZHOU, WEI TU, YUJIA XIA, HUANJUN HUANG and DE'AN TIAN \\ Department of Gastroenterology, Tongji Hospital, Tongji Medical College, Huazhong University of Science and Technology, \\ Wuhan, Hubei 430030 P.R. China
}

Received March 15, 2013; Accepted September 30, 2013

DOI: $10.3892 / \mathrm{ol} .2013 .1645$

\begin{abstract}
Astrocyte-elevated gene-1 (AEG-1) has been reported to be associated with cancer progression in various types of human cancers, including liver cancer. However, to date, the molecular mechanism of AEG-1 action on the growth of liver cancer cells has been poorly elucidated. The present study aimed to investigate the effect of AEG-1 on the proliferation and apoptosis of liver cancer cells and the role of IL-6 in this process using the HepG2 human hepatoma cell line. shRNAs targeting AEG-1 were used to silence the expression of AEG-1. The effects on cell growth were detected by 3-(4, 5-dimethylthiazol-2-yl)-2, 5-diphenyltetrazolium bromide, colony formation and cell cycle assays. Apoptosis was analyzed by flow cytometry. The expression of IL- 6 was examined by quantitative polymerase chain reaction and enzyme-linked immunosorbent assay, and the phosphorylation of Stat3 was detected by western blotting. AEG-1 knockdown was observed to induce cell proliferation inhibition and apoptosis through the suppression of IL-6 secretion. Stat3, a downstream target of IL-6 signaling, was suppressed in the AEG-1-silenced cells and target genes, including $\mathrm{Bcl}-2$ and crystalin, $\alpha \mathrm{B}$, which are associated with cell survival, were downregulated. Overall, the findings suggest that aberrant AEG-1 expression promotes the growth of HepG2 liver cancer cells, contributing to the progression of liver cancer, which may partly be mediated by IL-6 signaling.
\end{abstract}

\section{Introduction}

Liver cancer is one of the five most common types of cancer and a main cause for the mortality of patients with cancer (1). Liver cancer involves a rapidly growing tumor with a poor

Correspondence to: Professor De'an Tian, Department of Gastroenterology, Tongji Hospital, Tongji Medical College, Huazhong University of Science and Technology, 1095 Jiefang Avenue, Wuhan, Hubei 430030, P.R. China

E-mail: tjtiandean@126.com

Key words: astrocyte elevated gene-1, proliferation, apoptosis, IL-6, HepG2 cells prognosis (2). However, the effects of the main therapeutic methods, including liver transplantation and surgical resection, remain limited (3). Therefore, the investigation of tumor-related genes may provide benefits for new treatment options.

Astrocyte elevated gene-1 (AEG-1), also named MTDH or LYRIC, was first cloned as an HIV- and TNF- $\alpha$-inducible gene in primary human fetal astrocytes (9). Studies have suggested that AEG-1 expression was remarkably higher in glioma, breast, prostate, esophagus and liver cancers compared with that in the respective normal tissues (4-8). Previous studies have shown that AEG-1 synergizes with oncogenic Ha-ras to enhance the soft agar colony formation of non-tumorigenic immortalized melanocytes (10). Knockdown of AEG-1 induces apoptosis of prostate cancer cells through the downregulation of Akt activity and the upregulation of forkhead box 3 a activity (6). In addition, AEG-1 may promote the progression of hepatocellular carcinoma by activating $\mathrm{Wnt} / \beta$-catenin signaling (7). The data suggest that AEG-1 is able to induce growth promotion in liver cancer, but the molecular mechanism remains to be elucidated.

Gene silencing by RNA interference (RNAi) has become a powerful tool for functional genetic analyses due to the ability to create specific loss-of-function phenotypes. In the present study, RNAi was used to obtain the liver cancer HepG2 cell line with stably silenced expression of AEG-1. The effects of AEG-1 on proliferation and apoptosis were examined in the HepG2 cells. IL-6 is a significant inflammatory cytokine and play a key role in growth promotion in several human cancers, including biliary tract epithelial cancers, prostate cancer and multiple myeloma (11-12). IL-6 levels in patients with liver cancer are markedly higher than those in normal individuals (13). Furthermore, the high serum level of IL-6 has been shown to be closely associated with the progression of liver cancer (14). Data have suggested that IL-6 may activate several signaling pathways, including phosphoinositide 3-kinase, JAK/STAT and p38 mitogen-activated protein kinase by autocrine or paracrine pathways (15). Stat3, a key downstream target of IL-6 signaling, promotes the expression of several genes that are associated with cell survival, leading to tumor cell proliferation, apoptosis inhibition and an increased metastatic potential. The present study aimed to investigate the effects of AEG-1 on the proliferation and apoptosis of HepG2 cells, and explored the molecular mechanism of tumor growth in liver cancer. 


\section{Materials and methods}

Cell culture and AEG-1-knockdown cells. Human liver cancer Hep3B, HepG2, SMMC-7721, MHCC-97H, HCC-LM3 and SK-HEP-1 cell lines were cultured in Dulbecco's modified Eagle Medium (DMEM) with $10 \%$ fetal calf serum (Invitrogen Gibco, Carlsbad, CA, USA) and incubated at $37^{\circ} \mathrm{C}$ with $5 \%$ $\mathrm{CO}_{2}$. Two shRNA oligonucleotide duplexes against the AEG-1 sequence (NM_178812) were synthesized by GeneChem (Shanghai, China) according to the study by Yoo et al (7). The AEG-1 shRNAs were inserted into the psilencer2.0 vector. The successful plasmid construction was verified by DNA sequencing. The psilencer2.0-shAEG-1-1 and -2 and the psilencer 2.0 control vector, respectively, were transfected into semi-confluent HepG2 cells using Lipofectamine 2000 reagent (Invitrogen Gibco). After $24 \mathrm{~h}$, the transfected cells were trypsinized and replated into six-well plates (1:10), then selected for 14 days with $600 \mu \mathrm{g} / \mathrm{ml} \mathrm{G} 418$ to produce stable AEG-1-knockdown cell lines. A single colony of stable cells was selected for further culture and the concentration of G418 was subsequently reduced by half and maintained in cultivation.

3-(4,5-dimethylthiazol-2-yl)-2,5-diphenyltetrazolium bromide (MTT) assay and flow cytometry analysis. A total of $5 \times 10^{3}$ cells per well were plated into a 96-well plate. The cell growth was determined using the MTT assay with a wavelength of $550 \mathrm{~nm}$. For the apoptosis analyses, the cells were harvested and stained using a phycoerythrin-Annexin V apoptosis detection kit (BD Pharmingen, San Diego, CA, USA) according to the manufacturer's instructions. For the cell cycle analyses, the cells were harvested, washed twice with phosphate-buffered saline and fixed with precooled $75 \%$ ethanol. Prior to the flow cytometry analyses, the ethanol was removed and $500 \mu 1$ freshly made dying solution, containing $0.05 \mathrm{mg} / \mathrm{ml}$ propidium iodide and $0.025 \mathrm{mg} / \mathrm{ml}$ RNase, was added. Subsequent to being stained for $30 \mathrm{~min}$, the cells were subjected to flow cytometry analysis. A total of three replicates were performed.

Colony formation assay. The HepG2-shAEG-1 and HepG2-vector cells were trypsinized and replated into six-well plates at a density of $5 \times 10^{2}$ cells per well. After two weeks, the cells were washed twice with PBS and fixed with methanol/acetic acid (3:1; v:v) for $15 \mathrm{~min}$. The fixing solution was removed and the cells were stained with $0.2 \%$ crystal violet for $10 \mathrm{~min}$. The number of colonies was counted under a microscope (CKX41; Olympus, Tokyo, Japan).

Quantitative polymerase chain reaction ( $q P C R$ ). Total RNA was isolated and extracted from the cells using TRIzol reagent according to the manufacturer's instructions (Invitrogen). Total RNA $(0.5 \mu \mathrm{g})$ was used for cDNA synthesis with PrimeScript RT reagent kit (Takara, Dalian, China). qPCR, based on SYBR premix EX TAQ (Takara), was performed to quantify the mRNA levels on an ABI StepOne Real-Time PCR system (Applied Biosystems, Inc., Carlsbad, CA, USA). The value of $2^{-\triangle \mathrm{Ct}}$ was used to determine the fold changes between the samples. The sequences of the primers that were usedinthis study wereas follows: Forward,5'-CCTTGGGTCCA GTTGCCTTCT-3' and reverse, 5'-CCAGTGCCTCTTTGCTG
CTTTC-3' for IL-6; forward, 5'-CATGTGTGTGGAGAGCG TCCA-3' and reverse, 5'-GCCGGTTCAGGTACTCAGTCA-3' for Bcl-2; forward, 5'-TCGGAGAGCACCTGTTGGA-3' and reverse, 5'-CCATGTTCATCCTGGCGCTC-3' for crystalin, $\alpha \mathrm{B}$ (Cryab); and forward, 5'-GTTGCGTTACACCCTTTC TTG-3' and reverse, 5'-GACTGCTGTCACCTTCACCGT-3' for $\beta$-actin.

Western blot analysis. For the western blot analysis, the cells were lysed in RIPA buffer (Sigma, St Louis, MO, USA). The protein concentration was determined using a BCA Protein Assay kit (Pierce, Rockford, IL, USA). Total protein $(50 \mu \mathrm{g})$ was separated on a $10 \%$ SDS-PAGE gel and transferred to polyvinylidene difluoride membranes (Millipore, Bedford, MA, USA). The membranes were subsequently immunoblotted with primary antibody. Anti-AEG-1 antibody was purchased from Abcam (Cambridge, MA, USA) and anti-glyceraldehyde 3-phosphate dehydrogenase (GAPDH) was obtained from Epitomics, Inc. (Burlingame, CA, USA). Anti-p-Stat 3 and -Stat 3 antibodies were purchased from Cell Signaling Technology (Boston, MA, USA). A secondary goat anti-rabbit antibody solution (Santa Cruz Biotechnology Inc., Santa Cruz, CA, USA) was finally used for detection with an ECL kit (Pierce, Rockford, IL, USA), according to the manufacturer's instructions.

Enzyme-linked immunosorbent assay (ELISA) analysis. Semi-confluent HepG2-shAEG-1 and HepG2-vector cells were cultured in serum-free DMEM. The supernatants were collected after $48 \mathrm{~h}$, centrifuged at $210 \mathrm{x}$ g for $5 \mathrm{~min}$ at $4^{\circ} \mathrm{C}$ to remove the cellular debris, and stored at $-80^{\circ} \mathrm{C}$ until the analysis was performed by ELISA. Immunoreactive IL- 6 was quantified using the ELISA kit (R\&D Systems, Emeryville, CA, USA), according to basic laboratory instructions. Each data point represents readings from a minimum of four independent assays that were performed in triplicate.

Animal tumor model andxenograft.Eight male BALB/c-nu/nu mice (weight, $20 \mathrm{~g}$; age, four weeks old) were purchased from the animal center of Tongji Medical College, Huazhong University of Science and Technology (Wuhan, China). A total of five million HepG2-shAEG-1 and HepG2-vector cells in $0.1 \mathrm{ml}$ PBS each were injected subcutaneously into the right and left flank of each nude mouse. The length (L) and width (W) of the tumors were measured externally using a vernier caliper every week. The tumor volume was determined according to the equation: $\mathrm{V}=\left(\mathrm{LxW}^{2}\right) / 2$. The growth curve was drawn according to the change in tumor volume over time. The mice were sacrificed following after weeks, and the tumors were excised and analyzed by hematoxylin and eosin staining. All experiments were performed according to the guidelines of the local animal use and care committee.

Statistical analysis. The SPSS 16.0 software package (SPSS, Inc., Chicago, IL, USA) was used for the statistical analysis and measurement data are presented as the mean \pm standard deviation. The statistical significance of the differences was determined using Student's t-test. $\mathrm{P}<0.05$ was considered to indicate a statistically significant difference. 
A

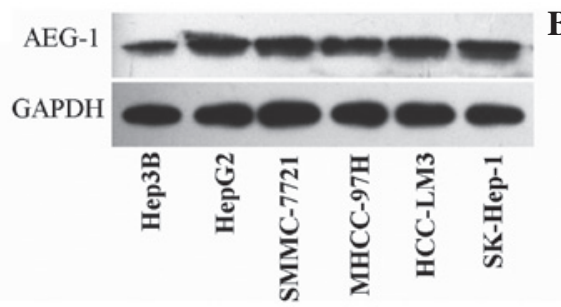

B

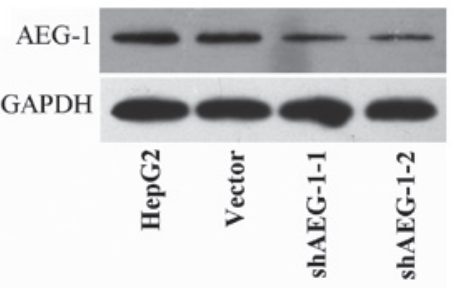

Figure 1. AEG-1 expression levels. (A) The expression of AEG-1 in six human liver cancer cell lines was analyzed by western blotting. (B) AEG-1 protein expression in AEG-1-silenced (HepG2-shAEG-1) and vector control (HepG2-vector) cells was detected by western blotting. GAPDH was used as a loading control. AEG-1, astrocyte-elevated gene-1; GAPDH, glyceraldehyde 3-phosphate dehydrogenase.

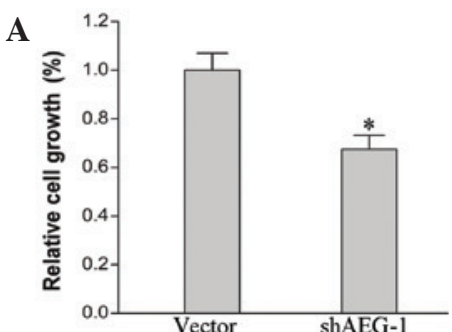

D

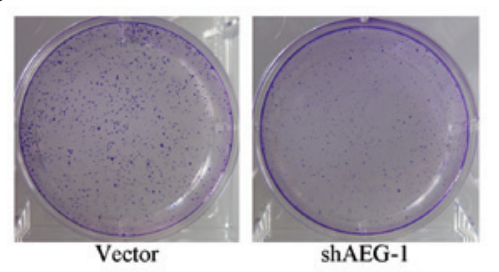

B

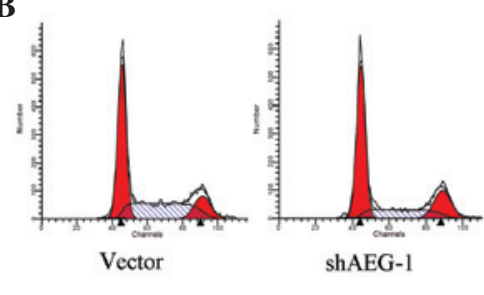

E

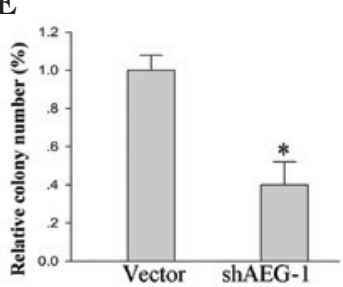

F

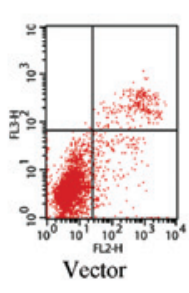

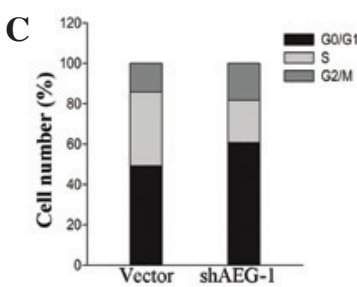

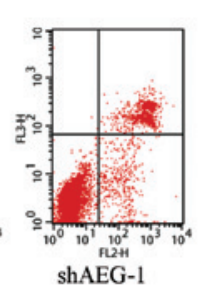

Figure 2. Effect of AEG-1 knockdown on the proliferation and apoptosis in HepG2 cells. (A) The proliferation of the AEG-1 knockdown and control cells was detected by MTT assay ( $\mathrm{P}<0.05)$. (B and C) FACS cell cycle analysis of AEG-1 knockdown cells and vector control. (D and E) Colony formation assay of the HepG2-shAEG-1 and HepG2-vector cells ("P<0.05). (F and G) Apoptosis analysis of the HepG2-shAEG-1 and HepG2-vector cells was detected by flow cytometry subsequent to staining the cells with Annexin V/PE ( $\left.{ }^{*} \mathrm{P}<0.05\right)$. Error bars represent SD, n=3 experiments. AEG-1, astrocyte-elevated gene-1; MTT, 3-(4, 5-dimethylthiazol-2-yl)-2, 5-diphenyltetrazolium bromide; FACS, fluorescence-activated cell sorting; PE, phycoerythrin.

\section{Results}

Establishing AEG-1-knockdown liver cancer cell lines. The protein expression of AEG-1 was assayed by western blotting in a panel of liver cancer Hep3B, HepG2, SMMC-7721, MHCC-97H, HCC-LM3, SK-Hep-1 cell lines. It was observed that the AEG-1 protein was overexpressed in all these liver cancer cell lines (Fig. 1A). The HepG2 cells exhibited high expression levels of AEG-1 and were selected for AEG-1 gene silencing. The psilencer2.0 (vector), psilencer2.0-shAEG-1-1 and 2 plasmids were stably transfected into the HepG2 cell line. Western blotting was used to detect the effect of AEG-1 silencing. Compared with the non-transfected HepG2 cells, the expression level of AEG-1 protein was remarkably inhibited in the HepG2-shAEG-1-1 and -2 cells. There was no significant difference between the HepG2-vector cells and the untreated HepG2 cells (Fig. 1B). The HepG2-shAEG-1-2 cells were chosen to complete the following experiments.

Knockdown of AEG-1 inhibits cell growth and promotes apoptosis in hepatoma HepG2 cells. To investigate the effects of AEG-1 on the growth of hepatoma HepG2 cells, cell cycle, cell proliferation and colony formation assays were performed. The cell cycle was significantly arrested in the HepG2-shAEG-1 cells. Compared with the vector control, the ratio of the cells in the $\mathrm{G}_{0} / \mathrm{G}_{1}$ phase was increased by $12 \%$ and the ratio of cells in the $\mathrm{S}$ phase was decreased by $16 \%$ in the HepG2-shAEG-1 cells (Fig. 2B and C). The cell proliferation ability was also inhibited by AEG-1 silencing. Knockdown of AEG-1 suppressed cell proliferation in the HepG2 cells by $26 \%$ (Fig. $2 \mathrm{~A} ; \mathrm{P}<0.05$ ). Furthermore, the ability of colony formation following AEG-1 silencing in the HepG2 cells was analyzed. The relative colony number was markedly decreased in the HepG2-shAEG-1 cells compared with the vector control (Fig. 2D and E; P<0.05). The knockdown of AEG-1-induced apoptosis by flow cytometry was examined in the HepG2 cells. Compared with the HepG2 cells that were transfected with empty vector plasmid, the apoptosis ratio increased significantly in the HepG2-shAEG-1 cells (Fig. 2F and G; P<0.05).

Knockdown of AEG-1 suppresses IL-6 secretion and inhibits Stat 3 activation. To determine whether IL-6 participates in the knockdown of AEG-1-induced growth inhibition and apoptosis, the expression of IL-6 in HepG2 cells was examined with AEG-1 silenced by shRNA. The mRNA level of IL-6 was decreased in the HepG2-shAEG-1 cells compared with that in the HepG2-vector cells (Fig. 3A; P<0.05). The concentration of IL- 6 in the culture supernatants of the HepG2-shAEG-1 and HepG2-vector cells was identified to be similar to the expression levels of IL- 6 mRNA. The secretion 

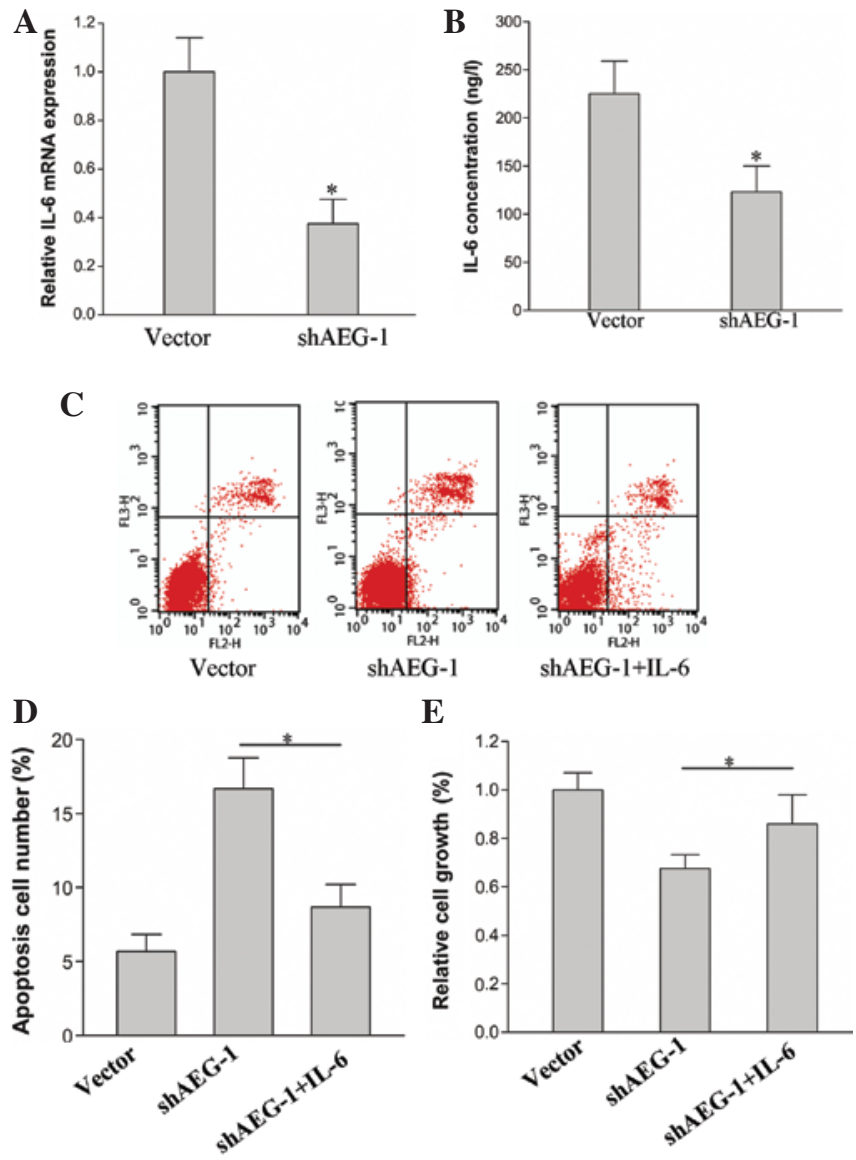

Figure 3. IL-6 expression in AEG-1 knockdown cells, and proliferation and apoptosis in HepG2-shAEG-1 cells treated with IL-6. (A and B) IL-6 expression was detected by qPCR and ELISA in the HepG2-shAEG-1 and HepG2-vector cells $\left({ }^{*} \mathrm{P}<0.05\right)$. (C and D) Apoptosis analysis of the HepG2-shAEG-1 cells that were treated with IL-6 (50 ng/l) was assayed by flow cytometry ( $\mathrm{P}<0.05)$. (E) The proliferation of the HepG2-shAEG-1 cells that were treated with IL-6 $(50 \mathrm{ng} / \mathrm{l})$ or remained untreated was detected by MTT assay $\left({ }^{*} \mathrm{P}<0.05\right)$. Error bars represent $\mathrm{SD}, \mathrm{n}=3$ experiments. AEG-1, astrocyte-elevated gene-1; qPCR, quantitative polymerase chain reaction; ELISA, enzyme-linked immunosorbent assay; MTT, 3-(4, 5-dimethylthiazol-2-yl)-2, 5-diphenyltetrazolium bromide.
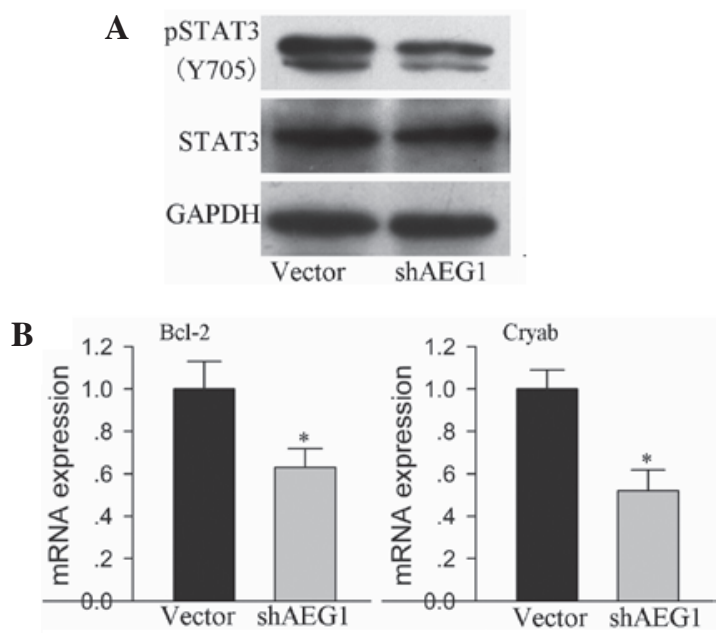

Figure 4. AEG-1 inhibition reduces activation of Stat3 signaling. (A) Stat3 expression levels were suppressed by AEG-1 silencing in HepG2 cells, as detected by western blotting. GAPDH was used as a loading control. (B) Stat3 target genes were downregulated in HepG2 cells with AEG-1 silencing, as detected by qPCR ( $\mathrm{P}<0.05)$. AEG-1, astrocyte-elevated gene-1; GAPDH, GAPDH, glyceraldehyde 3-phosphate dehydrogenase; qPCR, quantitative polymerase chain reaction; Cryab, crystalin, $\alpha \mathrm{B}$.
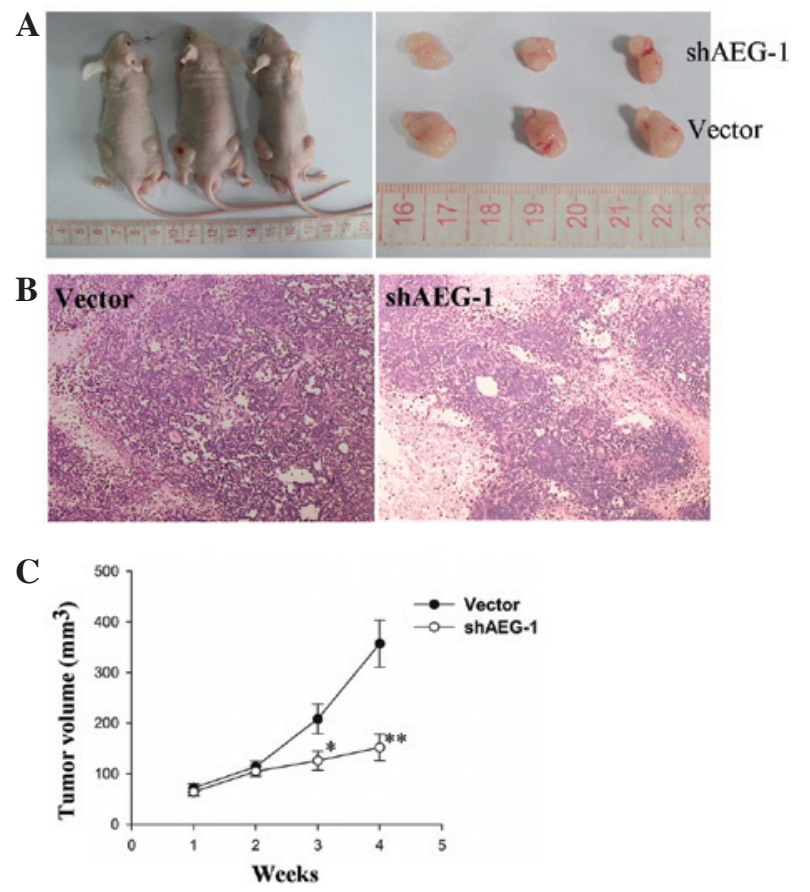

Figure 5. Knockdown of AEG-1 inhibits tumor growth. (A) Typical images of the mice and the tumors that were formed. (B) Hematoxylin and eosin staining of subcutaneous tumors in nude mice (magnification, x200). (C) Growth curve of tumor volumes ( $\mathrm{P}<0.05$ and $\left.{ }^{* *} \mathrm{P}<0.01\right)$.

of IL-6 in the HepG2-shAEG-1 cells was reduced compared with that of the HepG2-vector cells (Fig. 3B; P<0.05). In the subsequent experiments, the proliferation and apoptosis of the HepG2-shAEG-1 cells was analyzed following the treatment with IL-6 (50 ng/l). The results revealed that the proliferation ratio increased in the HepG2-shAEG-1 cells that were treated with IL-6 compared with that in the untreated cells (Fig. 3E; $\mathrm{P}<0.05)$. The apoptosis ratio decreased in the IL-6-treated cells (Fig. 3C and D; $\mathrm{P}<0.05$ ).

Stat3 is a significant transcription factor that is activated by IL-6 and associated cytokines. The present study demonstrated that AEG-1 inhibition was able to reduce the secretion of IL-6 in hepatoma HepG2 cells. Therefore, AEG-1 inhibition was investigated in order to determine whether it was able to downregulate Stat 3 phosphorylation. Stat 3 activation was observed to be suppressed in the HepG2 cells with shAEG-1 plasmid transfection, compared with that in the empty vector-transfected cells, which was confirmed by reduced tyrosine (Y705) phosphorylation of Stat3 (Fig. 4A). Furthermore, the expression of cell survival-related genes that are regulated by Stat 3 were identified in the AEG-1-silenced HepG2 cells. Bcl-2 and Cryab mRNA expression levels were reduced in the cells that were transfected with the shAEG-1 plasmid, compared with those in the mice that were transfected with the empty vector (Fig. 4B; P<0.05).

Knockdown of AEG-1 inhibits the growth of subcutaneous tumors in nude mice. Following subcutaneous cell inoculation in $\mathrm{BALB} / \mathrm{C}$ nude mice for seven days, the rate of tumor formation of the HepG2-shAEG-1 cells was $62.5 \%$ (5/8), whereas the tumor formation rate of the HepG2-vector cells was 75\% (6/8). Knockdown of AEG-1 in the HepG2 cells inhibited the growth of subcutaneous tumors and the tumor volumes were 
smaller than those in the mice that were inoculated with the HepG2-vector cells (Fig. 5A). The average tumor volume and growth rate in the HepG2-shAEG-1 cell inoculation group were markedly reduced compared with the vector control group (Fig. 5C; $\mathrm{P}<0.05$ and $\mathrm{P}<0.01$ ).

\section{Discussion}

The present study shows that the knockdown of AEG-1 may inhibit cell proliferation and promote apoptosis in the liver cancer HepG2 cell line, and the molecular mechanism may involve the suppression of IL- 6 secretion and the inhibition of Stat 3 activation.

Despite the fact that AEG-1 was first identified in 2002, studies on AEG-1 have increased gradually in the last five years and have mainly focused on the progression of tumors. Previous studies have suggested that AEG-1 is able to promote invasion and metastasis of hepatocellular carcinoma $(7,16,17)$. However, few studies have investigated the role of AEG-1 in the growth of liver cancer cells. Proliferation and apoptosis are two significant aspects during the tumor growth process (18). The poor prognosis of patients with liver cancer is mainly due to rapid growth and metastasis (2). Therefore, it is necessary to identify the association between tumor-related genes and the growth of liver cancer.

In the present study, the stable HepG2 cells were acquired with AEG-1 silencing. Inhibited proliferation, increased apoptosis and cell cycle arrest were observed in the HepG2-shAEG-1 cells. The tumor microenvironment is now considered to be a significant stimulative factor for tumors. Inflammatory cytokines, the main component of the tumor microenvironment, are closely associated with the development and progression of liver cancer (19). Our previous expression microarray analysis suggested that IL- 6 and IL-1 $\beta$ levels were markedly changed in AEG-1-overexpressed and-silenced HCC cells (unpublished data). The subsequent experiments demonstrated that IL-6 secretion was inhibited in AEG-1 knockdown cells, and exogenous IL-6 was able to reverse AEG-1 knockdown-induced anti-proliferation and apoptosis.

The IL-6 family of cytokines, including IL-6, ILF and oncostatin M, are known to be able to activate the Stat3 transcription factor (a downstream target of IL-6 signaling) through autocrine or paracrine pathways. Aberrant Stat 3 activation has been reported to exist in numerous human carcinomas (20-22). Upregulated phosphorylation of Stat 3 is closely associated with the promotion of growth and inhibition of apoptosis in tumor cells $(23,24)$. IL-6 is able to bind to IL-6R/gp130 and activate the Jak/Stat 3 pathway. IL-6 is a downstream regulator of AEG-1 in the HepG2 cells. The phosphorylation of Stat 3 was further investigated and it was observed that the activation of Stat 3 was reduced in the AEG-1-silenced cells. Furthermore, the cell survival-related genes, Bcl-2 and Cryab, which are regulated by Stat3, were downregulated in the AEG-1-silenced cells. Thus, the inhibition of proliferation and the promotion of apoptosis that was induced by AEG-1 silencing in the HepG2 cells was likely to be mediated by the inactivation of Stat 3 and the downregulated expression of Bcl-2 and Cryab. However, whether the molecular mechanism identified in the present study is functional in other liver cancer cells and other tumors requires further study.
In conclusion, the present study has demonstrated that AEG-1 plays a significant role in the proliferation and apoptosis of liver cancer HepG2 cells via downregulated IL-6 secretion and Stat 3 activation. Furthermore, AEG-1 knockdown inhibits the tumor growth in vivo. Therefore, this study provides an improved understanding of the role of AEG-1 in the growth of liver cancer.

\section{Acknowledgements}

This study was supported by grants from the National Science Foundation of China (no. 81070333) and the Natural Science Foundation of Hubei Province of China (no. 2012FFB02318).

\section{References}

1. Yang JD and Roberts LR: Hepatocellular carcinoma: a global view. Nat Rev Gastroenterol Hepatol 7: 448-458, 2010.

2. Pang RW, Joh JW, Johnson PJ, Monden M, Pawlik TM and Poon RT: Biology of hepatocellular carcinoma. Ann Surg Oncol 15: 962-971, 2008.

3. O'Neil BH and Venook AP: Hepatocellular carcinoma: the role of the North American GI Steering Committee Hepatobiliary Task Force and the advent of effective drug therapy. Oncologist 12: 1425-1432, 2007.

4. Brown DM and Ruoslahti E: Metadherin, a cell surface protein in breast tumors that mediates lung metastasis. Cancer Cell 5: 365-374, 2004.

5. Liu L, Wu J, Ying Z, Chen B, Han A, Liang Y, Song L, Yuan J, $\mathrm{Li} \mathrm{J}$ and $\mathrm{Li} \mathrm{M}$ : Astrocyte elevated gene-1 upregulates matrix metalloproteinase-9 and induces human glioma invasion. Cancer Res 70: 3750-3759, 2010.

6. Kikuno N, Shiina H, Urakami S, Kawamoto K, Hirata H, Tanaka Y, Place RF, Pookot D, Majid S, Igawa M and Dahiya R: Knockdown of astrocyte-elevated gene-1 inhibits prostate cancer progression through upregulation of FOXO3a activity. Oncogene 26: 7647-7655, 2007.

7. Yoo BK, Emdad L, Su ZZ, Villanueva A, Chiang DY, Mukhopadhyay ND, Mills AS, Waxman S, Fisher RA, Llovet JM, et al: Astrocyte elevated gene-1 regulates hepatocellular carcinoma development and progression. J Clin Invest 119: 465-477, 2009.

8. Yu C, Chen K, Zheng H, Guo X, Jia W, Li M, Zeng M, Li J and Song L: Overexpression of astrocyte elevated gene-1 (AEG-1) is associated with esophageal squamous cell carcinoma (ESCC) progression and pathogenesis. Carcinogenesis 30: 894-901, 2009.

9. Su ZZ, Kang DC, Chen Y, Pekarskaya O, Chao W, Volsky DJ and Fisher PB: Identification and cloning of human astrocyte genes displaying elevated expression after infection with HIV-1 or exposure to HIV-1 envelope glycoprotein by rapid subtraction hybridization, RaSH. Oncogene 21: 3592-3602, 2002.

10. Kang DC, Su ZZ, Sarkar D, Emdad L, Volsky DJ and Fisher PB: Cloning and characterization of HIV-1-inducible astrocyte elevated gene-1, AEG-1. Gene 353: 8-15, 2005.

11. Meng F, Yamagiwa Y, Taffetani S, Han J and Patel T: IL-6 activates serum and glucocorticoid kinase via p38alpha mitogen-activated protein kinase pathway. Am J Physiol Cell Physiol 289: C971-C981, 2005.

12. Kobayashi S, Werneburg NW, Bronk SF, Kaufmann SH and Gores GJ: Interleukin- 6 contributes to Mcl-1 up-regulation and TRAIL resistance via an Akt-signaling pathway in cholangiocarcinoma cells. Gastroenterology 128: 2054-2065; 2005.

13. Tilg H, Wilmer A, Vogel W, Herold M, Nölchen B, Judmaier G and Huber C: Serum levels of cytokines in chronic liver diseases. Gastroenterology 103: 264-274, 1992.

14. Matzaraki V, Alexandraki KI, Venetsanou K, Piperi C, Myrianthefs P, Malamos N, Giannakakis T, Karatzas S, Diamanti-Kandarakis E and Baltopoulos G: Evaluation of serum procalcitonin and interleukin-6 levels as markers of liver metastasis. Clin Biochem 40: 336-342, 2007.

15. Johnson C, Han Y, Hughart N, McCarra J, Alpini G and Meng F: Interleukin-6 and its receptor, key players in hepatobiliary inflammation and cancer. Transl Gastrointest Cancer 1: 58-70, 2012. 
16. Srivastava J, Siddiq A, Emdad L, et al: Astrocyte elevated gene-1 promotes hepatocarcinogenesis: novel insights from a mouse model. Hepatology 56: 1782-1791, 2012.

17. Sarkar D: AEG-1/MTDH/LYRIC in liver cancer. Adv Cancer Res 120: 193-221, 2013

18. Evan GI and Vousden KH: Proliferation, cell cycle and apoptosis in cancer. Nature 411: 342-348, 2001.

19. Leonardi GC, Candido S, Cervello M, Nicolosi D, Raiti F, Travali S, Spandidos DA and Libra M: The tumor microenvironment in hepatocellular carcinoma (review). Int J Oncol 40: 1733-1747, 2012

20. Walter M, Liang S, Ghosh S, Hornsby PJ and Li R: Interleukin 6 secreted from adipose stromal cells promotes migration and invasion of breast cancer cells. Oncogene 28: 2745-2755, 2009.
21. Lin MT, Lin BR, Chang CC, Chu CY, Su HJ, Chen ST, Jeng YM and Kuo ML: IL-6 induces AGS gastric cancer cell invasion via activation of the c-Src/RhoA/ROCK signaling pathway. Int J Cancer 120: 2600-2608, 2007.

22. Tu B, Du L, Fan QM, Tang Z and Tang TT: STAT3 activation by IL- 6 from mesenchymal stem cells promotes the proliferation and metastasis of osteosarcoma. Cancer Lett 325: 80-88, 2012.

23. Johnston PA and Grandis JR: STAT3 signaling: anticancer strategies and challenges. Mol Interv 11: 18-26, 2011.

24. Yu H and Jove R: The STATS of cancer - new molecular targets come of age. Nat Rev Cancer 4: 97-105, 2004. 